\title{
Adaptive monitoring of coral health at Scott Reef where data exhibit nonlinear and disturbed trends over time
}

\author{
Pubudu Thilan Abeysiri Wickrama Liyanaarachchige ${ }^{1}$, Rebecca Fisher ${ }^{2}$, Helen \\ Thompson $^{1}$, Patricia Menendez ${ }^{3}$, James Gilmour ${ }^{2}$, and James McGree ${ }^{1}$ \\ ${ }^{1}$ Queensland University of Technology \\ ${ }^{2}$ Australian Institute of Marine Science \\ ${ }^{3}$ Monash University Graduate School of Business
}

August 5, 2021

\begin{abstract}
Time series data are often observed in ecological monitoring. Frequently such data exhibit nonlinear trends over time potentially due to complex relationships between observed and auxiliary variables, and there may also be sudden declines over time due to major disturbances. This poses substantial challenges for modelling such data and also for model-based adaptive monitoring. We propose novel methods for finding adaptive designs for monitoring when historical data show such nonlinear patterns and sudden declines over time. This work is motivated by a coral reef monitoring program that has been established at Scott Reef; a coral reef off the Western coast of Australia. Data collected for monitoring the health of Scott Reef are considered, and semiparametric and interrupted time series modelling approaches are adopted to describe how these data vary over time. New methods are then proposed that enable adaptive monitoring designs to be found based on such modelling approaches. These methods are then applied to find future monitoring designs at Scott Reef and form a set of recommendations for future monitoring. Through applying the proposed methods, it was found that future information gain is expected to be similar across a variety of different sites, suggesting that no particular location needed to be prioritised at Scott Reef for the next monitoring phase. In addition, it was found that omitting some sampling sites/reef locations was possible without substantial loss in expected information gain, depending upon the disturbances that were observed. The resulting adaptive designs are used to provide recommendations for future monitoring in this region, and for reefs where changes to the current monitoring practices are being sought. Furthermore, as the methods used and developed throughout this study are generic in nature, this research has the potential to improve ecological monitoring more broadly where complex data are being collected over time.
\end{abstract}

\section{Hosted file}

Thilan et al 2021 Scott Reef monitoring .pdf available at https://authorea.com/users/429220/ articles/532960-adaptive-monitoring-of-coral-health-at-scott-reef-where-data-exhibitnonlinear-and-disturbed-trends-over-time 